\title{
Surgical Correction of Cryptotia with Superiorly Based Superficial Mastoid Fascia and Skin Paddle
}

\author{
Kotaro Yoshimura, M.D., Keiko Ouchi, M.D., Shinichi Wakita, M.D., Kouichi Uda, M.D., \\ and Kiyonori Harii, M.D.
}

Tokyo, Japan

An approach for the correction of cryptotia using a superiorly based superficial mastoid fascial flap and a skin paddle is introduced. The buried portion of the auricle was exposed through an incision made along the upper part of the helix, followed by an appropriate correction of the deformed cartilage. Protrusion of the upper portion of the auricle was accomplished using anchoring sutures. A small skin paddle was elevated from the caudal portion of the auricular sulcus with the superiorly based superficial mastoid fascia as the nutrient pedicle and transferred to the temporal skin defect. The procedure was performed in eight auricles in a total of seven patients with cryptotia. A satisfactory contour and protrusion of the auricle were maintained postoperatively, leaving the scar within the auricular sulcus. (Plast. Reconstr. Surg. 105: 836, 2000.)

Cryptotia is a congenital anomaly of the auricle frequently seen in Asian patients. In cryptotia, the upper third of the auricle is constricted and buried under the temporal skin. Cryptotia has been classified into two categories by Hirose et al. ${ }^{1}$ according to the type of cartilage constriction: type I (transverse muscle type or superior crus type) and type II (oblique muscle type or inferior crus type). Nonsurgical treatments are effective in infants under 6 months old, but surgical treatment is recommended in older patients. ${ }^{1-3}$ The deformity of the cartilage is properly corrected, and the skin deficiency in the upper third of the auricle is repaired. Although a simple Z-plasty or modification may be effective for mild examples of cryptotia, ${ }^{4}$ additional skin must be supplied to produce a well projected upper third of the auricle. Some methods use a triangular flap transposition from the temporal region, ${ }^{5-8}$ and others employ a rotation flap from the mastoid region. ${ }^{1,9}$ There are some reports of using a preauricular skin flap, ${ }^{10,11}$ and Mutimer and Mulliken ${ }^{12}$ used tissue expansion. Although the contours of corrected auricles were relatively satisfactory with conventional methods, a visible scar was left on the temporal hair-bearing area, the preauricular region, or the mastoid region. A modified skin-graft method ${ }^{13}$ leaves minimal scars only along the auricular sulcus and on the posterior surface of the auricles. We have developed a technique in which the skin defect is repaired with a skin paddle supplied by the underlying superficial mastoid fascial flap. This method leaves a scar along the invisible auricular sulcus and provides a wide exposure for correction of the deformed auricular cartilage.

\section{PATIENTS}

Our method was employed on eight auricles in a total of seven patients (Table I). Five patients had type I cryptotia, and the other two patients had type II. Six patients were female, and the ages varied from 1 year 5 months to 20 years (mean $\pm \mathrm{SD}, 7.0 \pm 7.4$ years). Patient 4 had undergone a surgical procedure without significant improvement before the first visit to our hospital.

\section{Operative Technigues}

\section{Design}

An incision line is made along the auricular sulcus while the buried portion of the auricle is 
TABLE I

Summarized Data of the Cases

\begin{tabular}{clclcc}
\hline Patient & Sex & $\begin{array}{c}\text { Age } \\
\text { (year) }\end{array}$ & Side & Type of Cryptotia & Complications \\
\hline 1 & Female & 2 & Bilateral & Type I & None \\
2 & Female & 15 & Tight & Type I & None \\
3 & Male & 2 & Type I & None \\
4 & Female & 20 & Type II & None \\
5 & Female & 5 & Typht I & None \\
6 & Female & 4 & Right & Type II & Epidermal necrosis \\
7 & Male & 1 & Tight & Type I & None \\
\hline
\end{tabular}

retracted with a skin hook. A skin paddle is designed over the caudal part of the auricular sulcus; two-thirds of the skin paddle should be taken from the mastoid region (Fig. 1, above, $l e f t$ ). The average size of the skin paddle is 30 $\mathrm{mm}$ long and $15 \mathrm{~mm}$ wide. For the design of
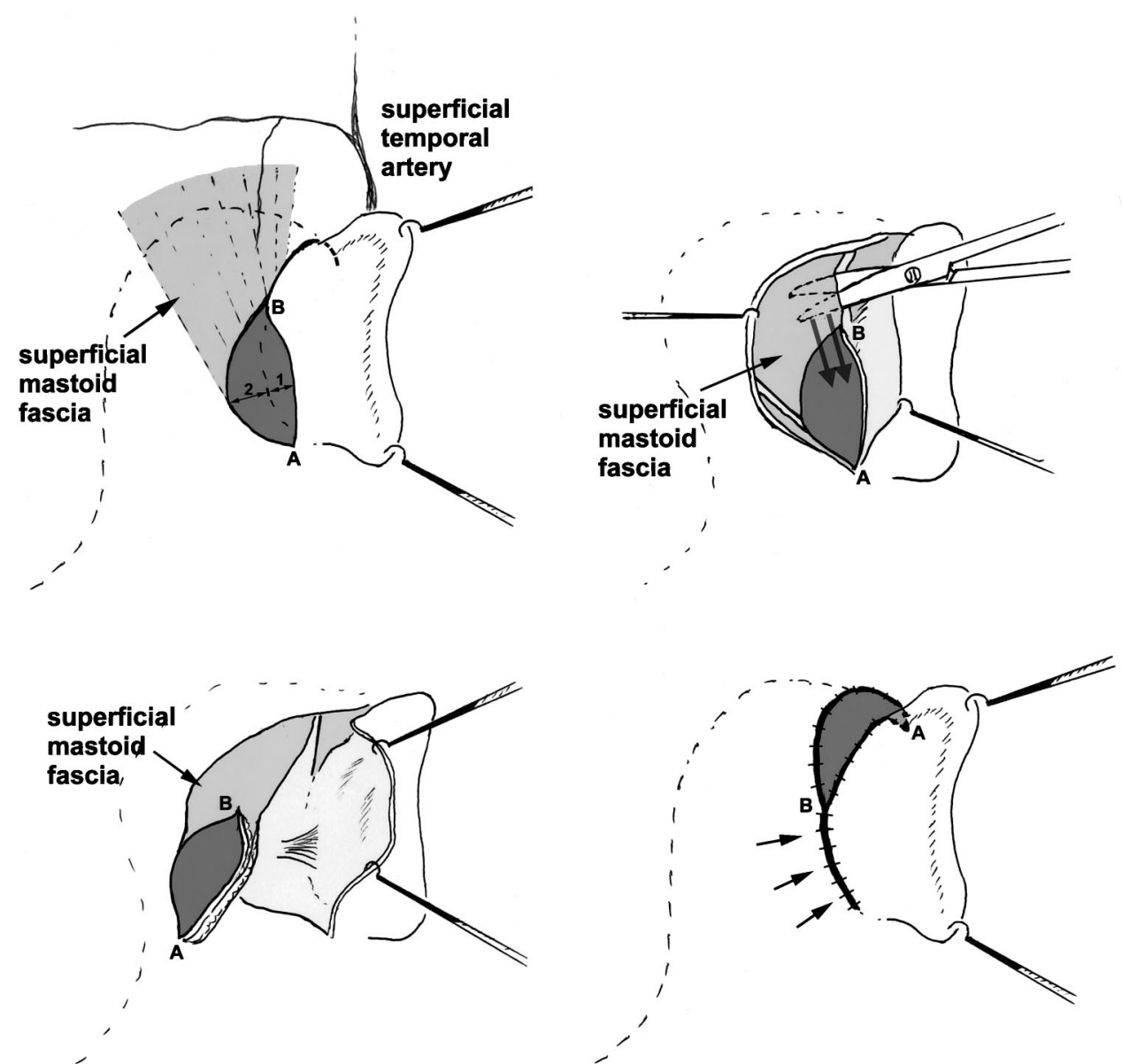

FIG. 1. Schema of operative procedure. (Above, left) Preoperative design. A skin paddle is designed on the caudal part of the auricular sulcus. Note that two-thirds of the skin paddle should be taken from the mastoid region. (Above, right) The undermining is made just deep to the superficial mastoid fascia and then extended inferiorly toward the skin paddle. (Below, left) The skin paddle and the superficial mastoid fascial flap are elevated. The skin paddle can be easily transposed to the temporal skin defect. (Below, right) After an appropriate correction of the cartilage deformity and transposition of the skin paddle, the mastoid skin is advanced anteriorly to the auricular sulcus, and the donor site is closed directly. 

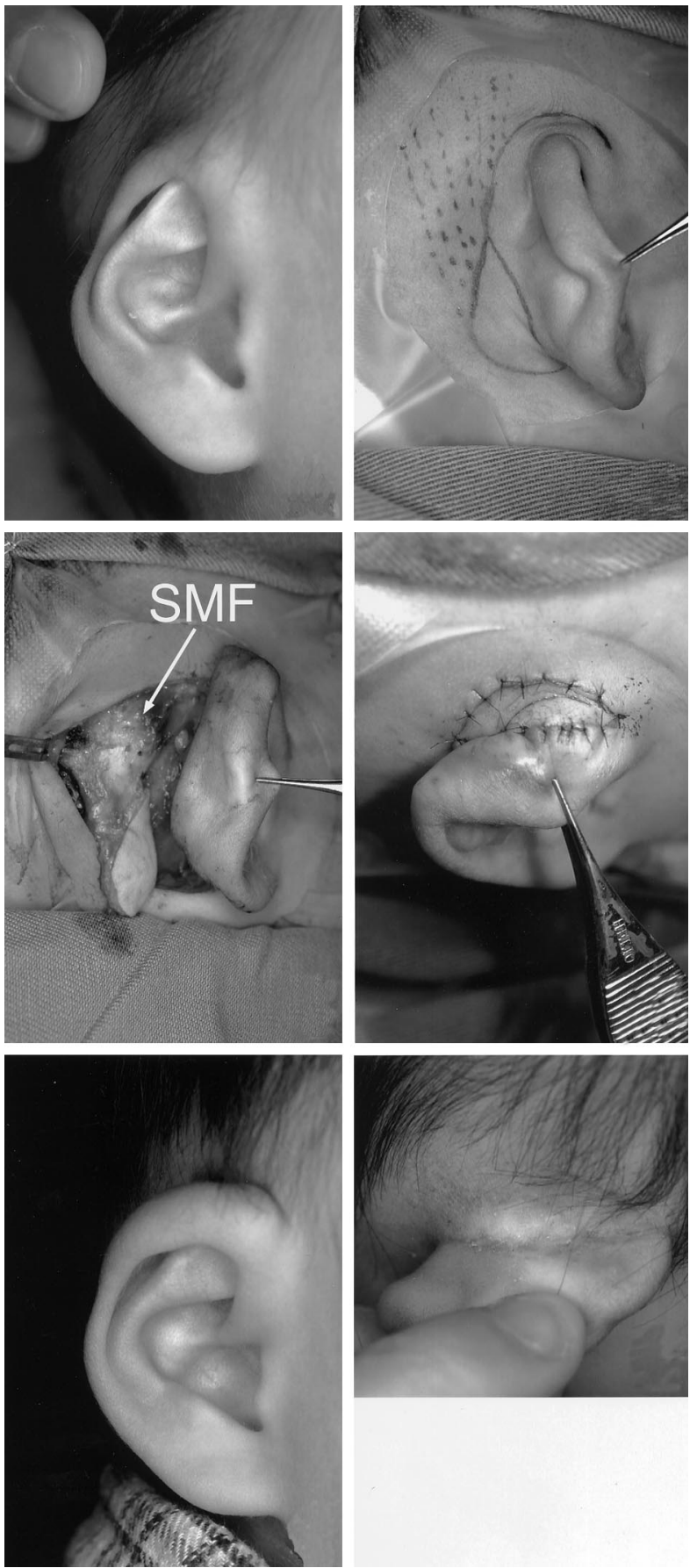

FIG. 2. Case 1 (patient 7): an 18-month-old boy with type I cryptotia. (Above, left) Preoperative view. (Above, right) Preoperative design. Note that more than a half of the skin paddle was designed on the mastoid region. (Center, left) The superficial mastoid fascial flap $(S M F)$ with a skin paddle was elevated. (Center, right) The skin flap was transposed to the temporal skin defect following correction of the constricted cartilage. (Below, left) A satisfactory contour and protrusion of the corrected auricle were preserved at postoperative 5 months. (Below, right) Five months later, the transposed skinpaddle was well preserved with minimal contraction. degrees from the perpendicular) to preserve the wide fascial pedicle (Fig. 1, above, left).

\section{Techniques}

A skin incision is made along the helix and around the skin paddle. After dissecting the posterior surface of the auricular cartilage inferiorly to the concha, the mastoid skin is carefully elevated superficial to the superficial mastoid fascia. Then, the anterior margin of the fascial flap is incised, and undermining is performed just deep to the superficial mastoid fascia. The undermining then is extended inferiorly toward the skin paddle (Fig. 1, above, right). After sufficient undermining of the superficial mastoid fascia, the skin paddle can be easily elevated. Finally, the posterior margin of the fascial flap is incised (Fig. 1, below, left). After an appropriate correction of the cartilage deformity, the conchal cartilage should be pulled cephalad and fixed to the temporal periosteum with a few absorbable stitches to enhance the protrusion of the upper part of the auricle. Then, the skin paddle is transposed and sutured to the temporal skin defect. Finally, the mastoid skin is advanced anteriorly to the auricular sulcus, and the donor site is directly closed (Fig. 1, below, right).

\section{RESULTS}

An extensive operative field for correction of the deformed cartilage was obtained by making a long incision along the auricular sulcus. The inferior tip (Fig. 1, point $A$ ) of the skin paddle was easily transferred to the anterior end of the auricular sulcus. Three representative cases were demonstrated in Figures 2 through 4 . Although the skin paddle often looked relatively pale just after the operation and partial epidermal necrosis was seen in patient 6 , the skin paddles survived in all cases. In patient 6 , more than half of the skin paddle was harvested from the posterior surface of the auricle, resulting in unstable vascularity from the underlying fascia (Fig. 3). Postoperative contraction of the skin flaps was minimal, and the contour of the corrected auricles was preserved very well. The scar on the retroauricular sulcus did not become hypertrophic in any cases. The present technique was successfully applied as the secondary operation in a patient who had undergone a previous surgical correction (Fig. 4). 

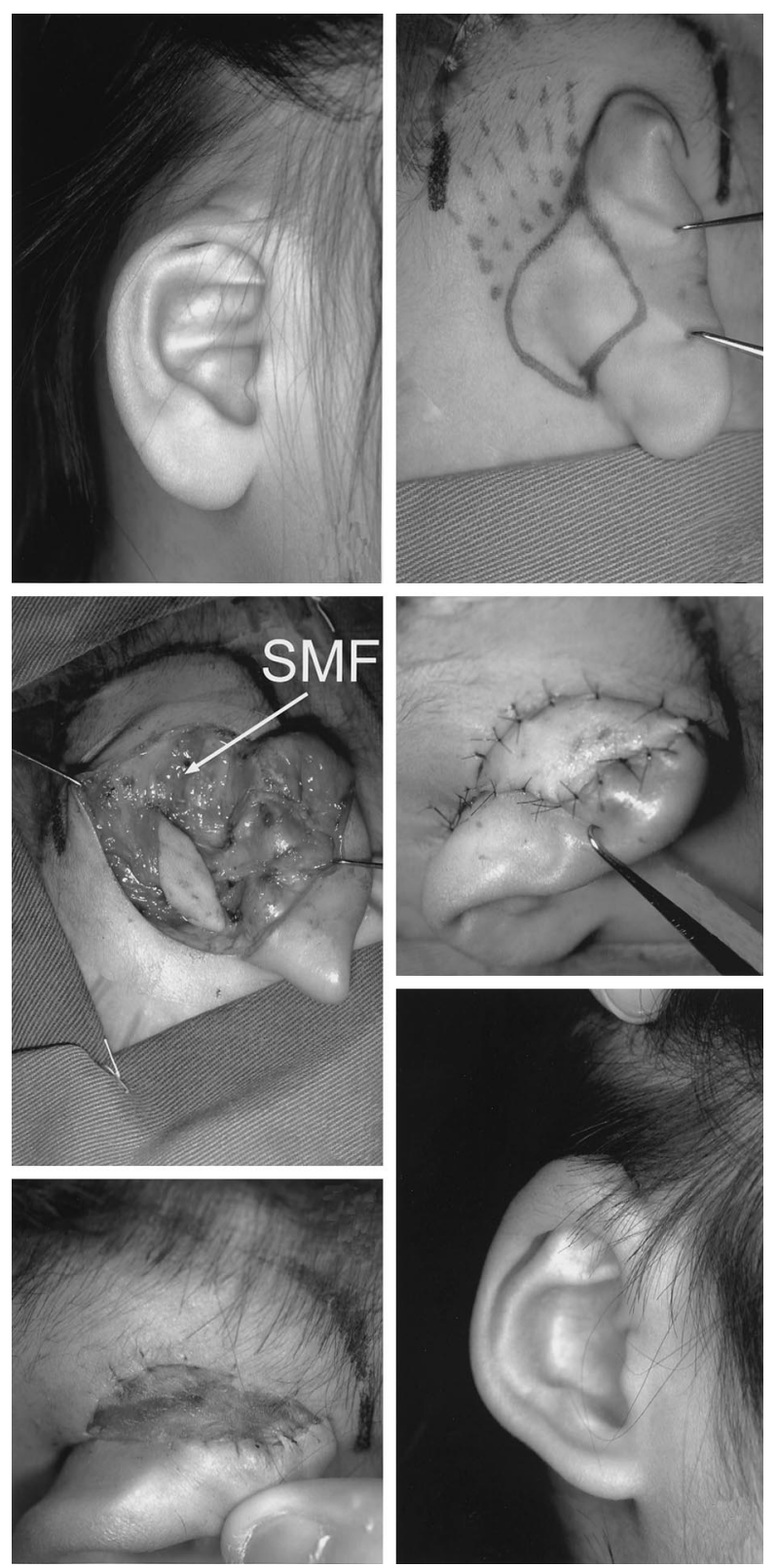

FIG. 3. Case 2 (patient 6): a 4-year-old girl with type II cryptotia. (Above, left) Preoperative view. (Above, right) Preoperative design. In this case, more than half of the skin paddle was designed on the posterior surface of the auricle. (Center, left) The superficial mastoid fascial flap $(S M F)$ with a skin paddle was elevated. A good exposure of the auricular cartilage was obtained. (Center, right) The skin flap was transposed to the temporal skin defect following correction of the constricted cartilage. The skin paddle appeared especially pale in this case. (Below, left) The transposed skin paddle showed epidermal necrosis 5 days after operation, but it completely survived. (Below, right) Six months later, satisfactory contour and protrusion of the corrected auricle were maintained.

\section{DisCUSSION}

The mastoid fascia is composed of two layers: the superficial mastoid fascia and the deep mastoid fascia. ${ }^{14}$ We used the superficial mastoid fascia, which corresponds cephalad to the superficial temporal fascia. The superficial mastoid fascia is primarily supplied by the posterior auricular artery and can be elevated as an anteroinferiorly based flap. ${ }^{14-16}$ This fascia is also supplied by (1) the posterior branch of the superficial temporal artery and/or the superior auricular artery ${ }^{17,18}$ and (2) the occipital artery. ${ }^{19}$ Thus, the superficial mastoid fascia can be elevated in three directions: as an anteroinferiorly based flap (using the posterior auricular artery), a posteroinferiorly based flap (using the occipital artery), or a superiorly based flap (using the superficial temporal artery). Among the three pedicles, the descending branch of the posterior branch of the superficial temporal artery, which is the nutrient vessel of the present fascial flap, is less reliable than the other two pedicles but has a sufficient blood supply to the present fascial flap and skin paddle.

There are some key points with regard to the operative design. The skin paddle of this technique is supplied by the underlying superficial mastoid fascia, although the perforating branch from the fascia has not been investigated. More than a half, preferably two-thirds, of the skin paddle should be taken from the mastoid region to make the vascularity of the skin paddle more reliable. Indeed, in case 6 , in which more than half of the skin paddle was harvested from the posterior surface of the auricle, epidermal necrosis of the skin paddle was observed. In addition, to preserve sufficient venous drainage of the skin flap, the fascial pedicle should be made as wide as possible.

Although a number of surgical methods have been introduced with relatively successful results, a visible scar is left on the temporal hair-bearing area, the preauricular region, or the mastoid region close to the hair line in most techniques. A modified skin-graft technique $^{13}$ leaves minimal scar formation on the posterior aspect of the auricle and the auricular sulcus, although it has some drawbacks (e.g., the time required for operation and problems with wound healing). Nakajima et al. ${ }^{20}$ described a method using a subcutaneous pedicled flap from the mastoid region. How- 

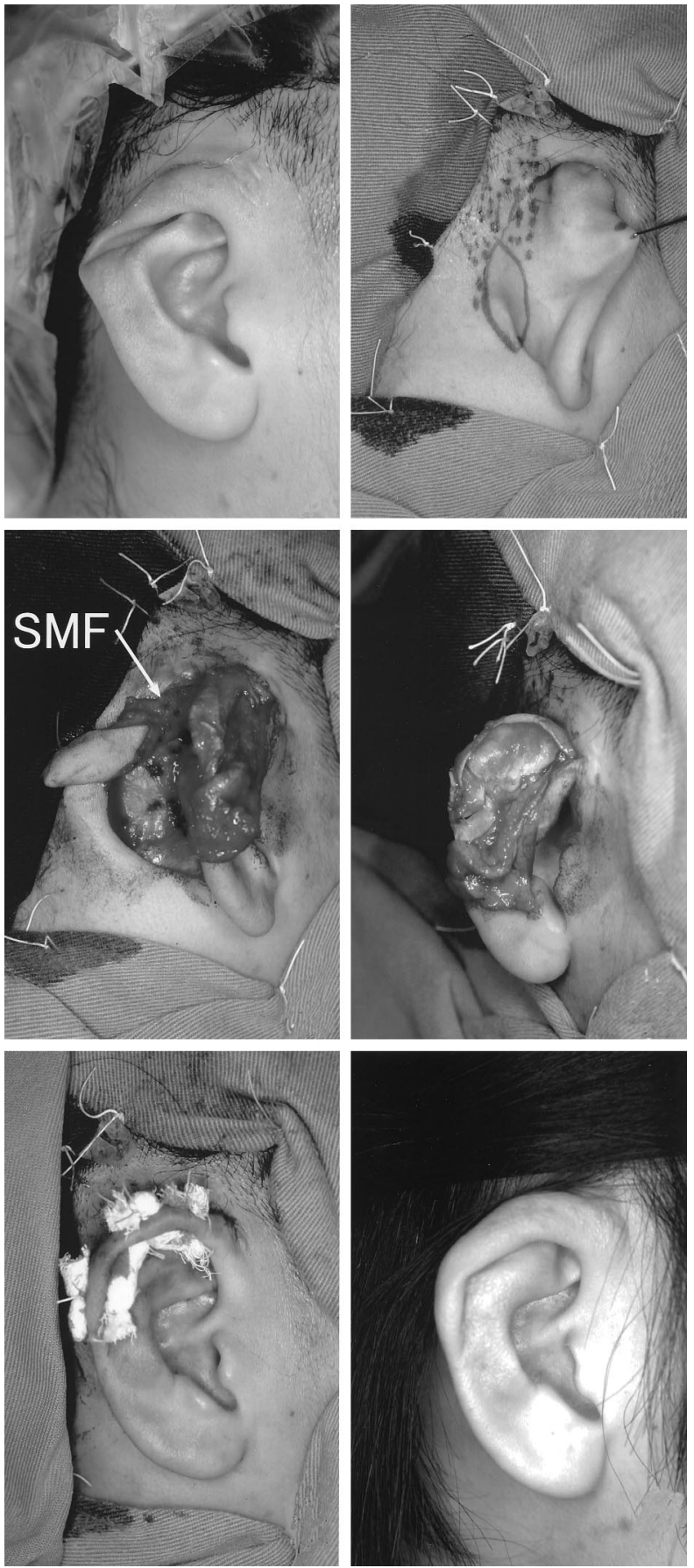

FIG. 4. Case 3 (patient 4). A 20-year-old woman had undergone surgical correction of type II cryptotia at another hospital without significant improvement 1 year before the first visit to our hospital. (Above, left) Preoperative view. (Above, right) Preoperative design. (Center, left) The superficial mastoid fascial flap $(S M F)$ with a skin paddle was elevated, and the upper half of the auricular cartilage was exposed. (Center, right) The deformity of the cartilage was properly repaired. A frontal view. (Below, left) The skin paddle was transposed, and the donor site was closed. Just after the surgery, some bolster fixations were performed along the repaired helix. (Below, right) Six months later, satisfactory contour of the corrected auricle was maintained. ever, the skin flap is supplied by subcutaneous tissue and elevated supraperiosteally. The dissection of the pedicle was performed minimally, especially in the anterior region, to preserve the vascularity, resulting in restricted mobility of the flap and distortion of the pedicle. The present authors propose a new technique for surgical correction with a skin flapthe vascularity of which is based on the underlying superficial mastoid fascia. This technique provides relatively reliable vascularity, sufficient mobilization of the skin flap, and wide exposure of the auricular cartilage, leaving a scar only on the invisible postauricular region along the auricular sulcus.

Kotaro Yoshimura, M.D.

Department of Plastic, Reconstructive, and Aesthetic Surgery

University of Tokyo

7-3-1, Hongo, Bunkyo-Ku

Tokyo, 113-8655, Japan

yoshimura-pla@h.u-tokyo.ac.jp

\section{REFERENCES}

1. Hirose, T., Tomono, T., Matsuo, K., et al. Cryptotia: Our classification and treatment. Br. J. Plast. Surg. 38: 352, 1985.

2. Anze, M., Shioya, N., and Akiyama, T. Bloodless treatment of cryptotia. Keisei Geka 14: 263, 1971.

3. Matsuo, K., Hayashi, R., Kiyono, M., et al. Nonsurgical correction of congenital auricular deformities. Clin. Plast. Surg. 17: 383, 1990.

4. Yanai, A., Tange, I., Bandoh, Y., et al. Our method of correcting cryptotia. Plast. Reconstr. Surg. 82: 965, 1988.

5. Kubo, I. Taschenohres und Otoplastik. Otolaryngology (Tokyo) 6: 105, 1933.

6. Fukuda, O. Otoplasty of cryptotia. Keisei Geka 11: 117, 1968.

7. Washio, H. Cryptotia: Pathology and repair. Plast. Reconstr. Surg. 52: 648, 1973.

8. Ono, I., Gunji, H., Suda, K., et al. A new operative method for treating severe cryptotia. Plast. Reconstr. Surg. 96: 461, 1995.

9. Onizuka, T., Tokunaga, S., and Yamada, K. A method for repair of cryptotia. Plast. Reconstr. Surg. 62: 734, 1978.

10. Elsahy, N. I. An alternative technique for correction of cryptotia. Ann. Plast. Surg. 23: 66, 1989.

11. Chana, J. S., and Fourie, L. R. A technique for the correction of cryptotia. Ann. Plast. Surg. 39: 261, 1997.

12. Mutimer, K. L., and Mulliken, J. B. Correction of cryptotia using tissue expansion. Plast. Reconstr. Surg. 81: 601, 1988.

13. Park, S., Takushima, M., and Minegishi, M. Reconstruction of cryptotia using a skin graft. Ann. Plast. Surg. 32: 441, 1994.

14. Park, C., Lee, T. J., Shin, K. S., and Kim, Y. W. A singlestage two flap method of total ear reconstruction. Plast. Reconstr. Surg. 88: 404, 1991. 
15. Yoshimura, K., Nakatsuka, T., Ichioka, S., et al. Onestage reconstruction of the upper part defect of the auricle. Aesthetic Plast. Surg. 22: 352, 1998.

16. Yoshimura, K., Asato, H., Nakatsuka, T., et al. Elevation of a constructed auricle using the anteriorly-based mastoid fascial flap. Br. J. Plast. Surg., 52: 530, 1999.

17. Kobayashi, S., Yoza, S., Kakibuchi, M., et al. Retroauricular hairline flap transfer to the face. Plast. Reconstr. Surg, 96: 42, 1995.
18. Song, R., Song, Y., Qi, K., et al. The superior auricular artery and retroauricular arterial island flaps. Plast. Reconstr. Surg. 98: 657, 1996.

19. Sharma, R. K., Kobayashi, K., Jackson, I. T., et al. Vascular anatomy of the galeal occipitalis flap: A cadaver study. Plast. Reconstr. Surg. 97: 25, 1996.

20. Nakajima, T., Yoneda, K., and Yoshimura, Y. Correction of cryptotia using a subcutaneous pedicled flap. Br. J. Plast. Surg. 44: 406, 1991. 